\title{
Research on UWB Precise Indoor Positioning in Ship Based on Intuitionistic Fuzzy
}

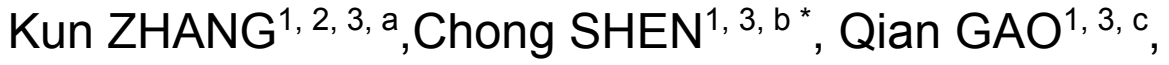 \\ Hai-feng WANG ${ }^{1,2, d}$, Yu-fei WANG ${ }^{1, e}$
}

1. State Key Laboratory of Marine Resources Utilization in South China Sea, Hainan University, Haikou, Hainan, 570228, China

2. College of Ocean Information Engineering, Hainan Tropical Ocean University, Sanya, Hainan, 572022, China

3. College of Information Science and Technology, Hainan University, Haikou, Hainan, 570228, China

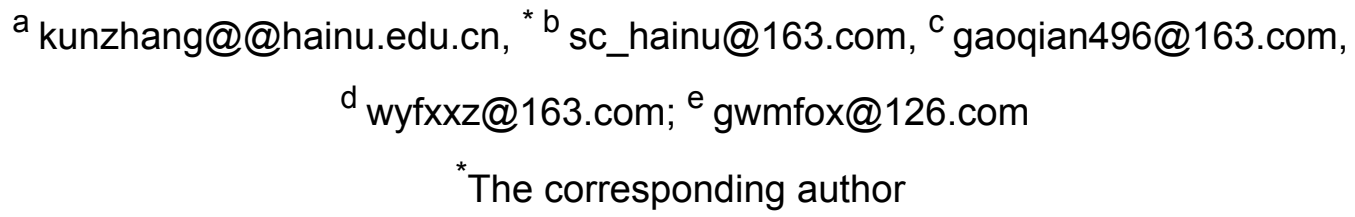

Keywords: Intuitive blur; Similarity measures; UWB; Accurate positioning in the room.

Abstract. Based on the related generalization of Vague set algorithm of intuitionistic fuzzy theory, this paper applies it to UWB marine ship precision positioning technology, puts forward new research methods and calculation formulas, and constructs the similarity measure between them and obtains new formula method So that the next step in the OMNET ++ network simulation platform to verify the performance of related algorithms, and simulation as an auxiliary means, the actual combination of Hainan EVK 1.0 and follow-up hardware platform testing and implementation.

\section{Introduction}

The ship is the main element of the maritime traffic system, widely used in commercial and military marine transport activities and even combat operations. The development trend of modern maritime ships is functional diversification, bulky, complex structure, its internal wireless positioning coverage of the ship's structural environment monitoring, personnel location monitoring and wireless transmission and so on is of great significance. The wireless positioning system monitors the target in real time by measuring the physical characteristics of the radio waves in the specified space. With the development of industrial 4.0 standard and the continuous development of Internet of Things technology, the application of real-time positioning of personnel, goods, assets and equipment in various industries is increasing. At the same time, security monitoring, national defense aerospace, unmanned operation, Sea area exploration, digital tourism and other emerging areas of real-time location system (RTLS) demand is also growing.

\section{Analysis of Theory and Present Situation}

For different needs, academia and industry put forward a variety of positioning technology, the technology in the applicable space, positioning accuracy and so there is a big difference, as shown in Figure 1. 


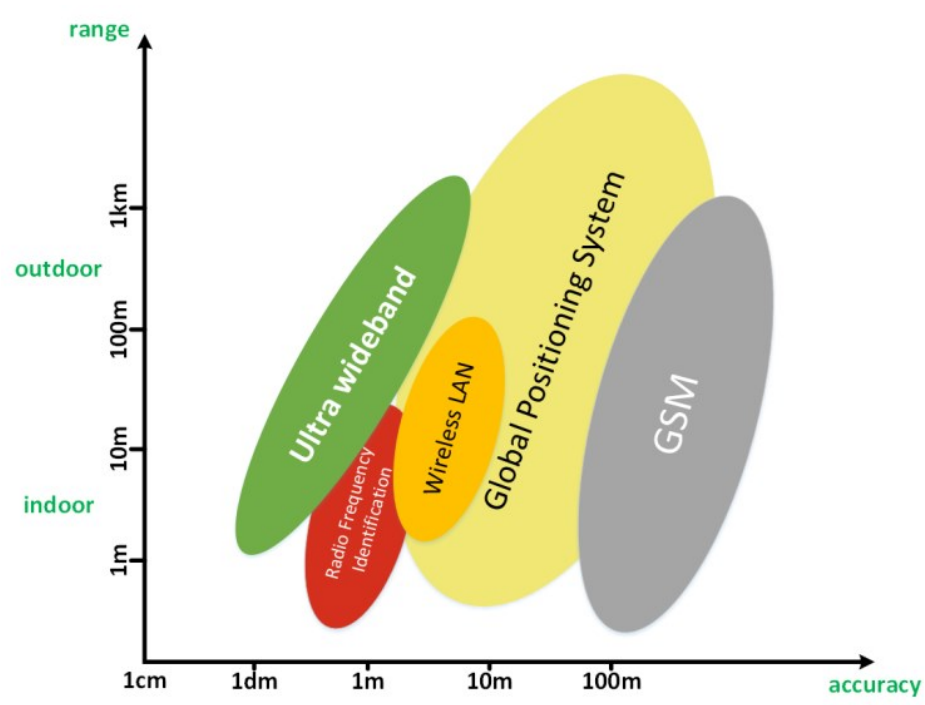

Figure 1. Comparison of characteristics of various location techniques

Global Positioning System (GPS), a universal solution for outdoors positioning, has a general positioning accuracy of above $10 \mathrm{~m}$-level without the help of auxiliary means. While for indoor positioning, the various positioning solutions based on traditional radiotechnics remains unsatisfactory, as the indoor signal propagation environment is more complicated than the outdoor environment, especially in indoors of maritime ship, which is also influenced by metallic materials and local spatial masking, making it a challenge to accurately analyze the parameters such as arrival time and arrival angle of signals [1]. The wireless local area networks positioning positions according to signal intensity, and is influenced by channel disturbance, with positioning accuracy unable to be guaranteed even after channel is estimated or compensated, hence the accuracy is generally meter-level; and its relatively small band width makes the received power disturbance produce large deviation for the positioning accuracy [2]. The radio frequency recognition and Bluetooth positioning is characterized by low power dissipation, small time lag and low cost, etc. yet with lower transmission rate, hence it is not applicable to large data transmission scene. Another drawback of this technology is its meter-level positioning accuracy, and proneness to disturbance of noise signal, leading to short transmission range [3]. Similar to Bluetooth, $2.4 \mathrm{GHz}$ wireless sensor network waveband positioning is also largely influenced by environment, leading to data inaccuracy when there are obstacles or interference signal of electromagnetic wave.

Relative to other positioning modes, IEEE 802.15.4a ultra wideband (UWB) baseband applied to positioning has absolute advantages in terms of accuracy, real-time performance and data band width, etc., with real-time response frequency up to $10 \sim 40 \mathrm{~Hz}$, compared with generally below $1 \mathrm{~Hz}$ for other location techniques The pulsewidth of impulse radio ultra-wideband (IR-UWB) is only nanosecond level or subnanosecond level. The response frequency and pulsewidth make positioning accuracy of UWB able to achieve centimeter level in the abstract. With excellent antimultipath capacity, high time resolution and certain penetrating power, UWB technology can meet the demands for real time positioning and dynamic data collection in complicated indoor multipath environment. The large-scale node pinpointing in broad scope can be realized via expanding of IEEE 802.15.4a UWB network itself or combining sensor network or heterogeneous networking of wireless local area networks.

Relative to other positioning modes, IEEE 802.15.4a ultra wideband (UWB) baseband applied to positioning has absolute advantages in terms of accuracy, real-time performance and data band width, etc., with real-time response frequency up to $10 \sim 40 \mathrm{~Hz}$, compared with generally below $1 \mathrm{~Hz}$ for other location techniques The pulsewidth of impulse radio ultra-wideband (IR-UWB) is only nanosecond level or subnanosecond level. The response frequency and pulsewidth make positioning accuracy of UWB 
able to achieve centimeter level in the abstract. With excellent antimultipath capacity, high time resolution and certain penetrating power, UWB technology can meet the demands for real time positioning and dynamic data collection in complicated indoor multipath environment. The large-scale node pinpointing in broad scope can be realized via expanding of IEEE 802.15.4a UWB network itself or combining sensor network or heterogeneous networking of wireless local area networks.

In 1986, Atanassov raised the theory of intuitionistic fuzzy set [4], and Gao and Buehrer raised the theory of Vague set [5] in 1993, which are all generalization of fuzzy set [6] theory. Besides, Bustince and Burilbo proved that Vague set is intuitionistic fuzzy set [7]. Furthermore, Deschrijver and Kerre [8] proved interval value fuzzy set and intuitionistic fuzzy set are essentially same. Compared with fuzzy set which only considers the concept of membership degree, the intuitionistic fuzzy set considers the concepts of the membership degree, non-membership degree and hesitancy degree at the same time. The intuitionistic fuzzy set describes fuzzy message in a more comprehensive, detailed and visual way. Space is a measure of intuitionistic fuzzy set and an important constituent of intuitionistic fuzzy set theory.

Especially it is applied to a good many aspects such as intuitionistic fuzzy reasoning, intuitionistic fuzzy pattern recognition, intuitionistic fuzzy multiple attribute decision making, etc. Therefore, range formulas are constantly raised by people. Therefore, the paper improves this range formula to be applied to UWB precise indoors positioning in ship. The paper improved formula calculation method using distance measure definition of intuitionistic fuzzy set to make the measuring indices of positioning accuracy more precise.

\section{Range Formula of Existing Intuitionistic Fuzzy Set}

Review of range formula of existing intuitionistic fuzzy set. In the following, generally set $A=\left\{\left\langle x_{i}, \mu_{A}\left(x_{i}\right), v_{A}\left(x_{i}\right): x_{i} \in X\right\rangle\right\}, B=\left\{\left\langle x_{i}, \mu_{B}\left(x_{i}\right), v_{B}\left(x_{i}\right): x_{i} \in X\right\rangle\right\}$ represents intuition fuzzy set on limited domain of discourse $X$. Only took following 4 range formulas of intuitionistic fuzzy set as representatives.

Szmidt E and Kacprzyk raised the distance measure of intuitionistic fuzzy set on limited domain of discourse $X$ in literature [9].

$$
\begin{aligned}
& D_{1}(A, B)=\frac{1}{2 n} \sum_{i=1}^{n} \mid\left(\mu_{A}\left(x_{i}\right)-\mu_{B}\left(x_{i}\right)\right)+\left(v_{A}\left(x_{i}\right)-v_{B}\left(x_{i}\right)+\left(\pi_{A}\left(x_{i}\right)-\pi_{B}\left(x_{i}\right) \mid\right.\right. \\
& D_{2}(A, B)=\sqrt{\frac{1}{2 n} \sum_{i=1}^{n}\left[\left(\mu_{A}\left(x_{i}\right)-\mu_{B}\left(x_{i}\right)\right)^{2}+\left(v_{A}\left(x_{i}\right)-v_{B}\left(x_{i}\right)\right)^{2}+\left(\pi_{A}\left(x_{i}\right)-\pi_{B}\left(x_{i}\right)\right)^{2}\right]}
\end{aligned}
$$

Szmidt E and Kacprzyk raised the distance measure of intuitionistic fuzzy set on limited domain of discourse $X$ in literature [10].

$$
\begin{aligned}
& D_{3}(A, B)=\frac{1}{n} \sum_{i=1}^{n} \max \left\{\mid\left(\mu_{A}\left(x_{i}\right)-\mu_{B}\left(x_{i}\right)|,|\left(v_{A}\left(x_{i}\right)-v_{B}\left(x_{i}\right) \mid\right\}\right.\right. \\
& D_{4}(A, B)=\sqrt{\frac{1}{n} \sum_{i=1}^{n} \max \left\{\left(\mu_{A}\left(x_{i}\right)-\mu_{B}\left(x_{i}\right)\right)^{2},\left(v_{A}\left(x_{i}\right)-v_{B}\left(x_{i}\right)\right)^{2}\right\}}
\end{aligned}
$$

Existing definition of distance measure of intuitionistic fuzzy set. $X u$ raised the existing definition of intuitionistic fuzzy set in literature [10]

Definition $4(\mathrm{Xu}[10])$ Set $A, B, C \in \operatorname{IFS}(X)$, mapping $D: \operatorname{IFS}(X) \times \operatorname{IFS}(X) \rightarrow[0,1]$ is called the distance measure of intuitionistic fuzzy set $\mathrm{A}$ and $\mathrm{B}$. If they meet following properties:

(P1) $D(A, B)=D(B, A)$;

(P2) $D(A, B)=0$ and $A=B$; 
(P3) $0 \leq D(A, B) \leq 1$;

(P4) If $A \subseteq B \subseteq C, D(A, C) \geq D(A, B) \vee D(B, C)$.

Formula (1)-(9) meet properties $(P 1)-(P 4)$

Reflection on the existing definition of distance measure of intuitionistic fuzzy set. Since existing distance measure formulas (1)-(4) meet properties $(P 1)-(P 4)$, it is necessary to examine definition 5 . It is obvious that the properties $(P 1),(P 3),(P 4)$ in definition 5 are specific embodiment of the basic properties of classical distance measure in intuistionistic fuzzy set, while as the property of boundary conditions of distance in intuitionistic fuzzy set, $(P 2)$ should be put more focus in.

Here designed 5 groups of data, as shown in table 1 and analyzed formulas (1)-(9). When $x_{j}=y_{j}=\left\langle x_{j}, \mu\left(x_{j}\right), v\left(x_{j}\right)\right\rangle, j=1,2,3,4,5$, used formulas (1)-(4) to calculate, all had following result figured out:

$$
D_{i}\left(x_{j}, y_{j}\right)=0,(j=1,2, \cdots \quad 2,3,4) \text { 。 }
$$

Although the general way of thinking is "the distance between self and self is the nearest". However, the particularity of intuitionistic fuzzy set renders situation not so simple. A close analysis on expression (10) shows that some cases are contradicting with people's intuitionistic knowledge and are open to discussion.

Table 1 Table of data of 5 groups of intuitionistic fuzzy set

\begin{tabular}{llllll}
\hline$x_{j}$ & $\left\langle x_{1}, 1,0\right\rangle$ & $\left\langle x_{2}, 0,1\right\rangle$ & $\left\langle x_{3}, 0.3,0.7\right\rangle$ & $\left\langle x_{4}, 0.4,0.3\right\rangle$ & $\left\langle x_{5}, 0,0\right\rangle$ \\
\hline$y_{j}$ & $\left\langle y_{1}, 1,0\right\rangle$ & $\left\langle x_{2}, 0,1\right\rangle$ & $\left\langle x_{3}, 0.3,0.7\right\rangle$ & $\left\langle x_{4}, 0.4,0.3\right\rangle$ & $\left\langle x_{5}, 0,0\right\rangle$ \\
\hline
\end{tabular}

In expression (10), $D_{i}\left(x_{1}, y_{1}\right)=D_{i}\left(x_{2}, y_{2}\right)=0,(i=1,2,3,4)$, which accords with people's intuition, as for common intuitionistic fuzzy value $<\mathrm{x} 1,1,0>$ and $<\mathrm{x} 2,0,1>$, not only $\mu\left(x_{j}\right)=\mu\left(y_{j}\right), v\left(x_{j}\right)=v\left(y_{j}\right)$, $\mathrm{j}=1,2$; and $\pi_{x_{j}}=\pi_{y_{j}}=0, j=1,2$, i.e. for common intuitionistic fuzzy value $<\mathrm{x} 1,1,0>$ and $<\mathrm{x} 2,0,1>$, the reasonable value for distance between self and self is the minimal value of 0 .

$D_{i}\left(x_{3}, y_{3}\right)=0,\left(i=1,2, \cdots \quad\right.$, this is also normal, as for fuzzy value $x_{3}$ and $y_{3}$, not only $\mu\left(x_{3}\right)=\mu\left(y_{3}\right), v\left(x_{3}\right)=v\left(y_{3}\right)$, and $\pi_{x_{3}}=\pi_{y_{3}}=0$, i.e. for fuzzy value, the reasonable value for distance between self and self is also the minimal value of 0

$d_{i}\left(x_{4}, y_{4}\right)=0, i=(1,2, \cdots \quad, \quad$ this is contradicting with people's knowledge, as $x_{4}=\left\langle x_{4}, 0.4,0.3\right\rangle==y_{4}=\left\langle y_{4}, 0.4,0.3\right\rangle$, it is a true intuitionistic fuzzy value, not only $\mu\left(x_{4}\right)=\mu\left(y_{4}\right)=0.4, v\left(x_{4}\right)=\nu\left(y_{4}\right)=0.3$, but also $\pi_{x_{3}}=\pi_{y_{3}}=0.3 \neq 0$. The characteristic of true intuitionistic fuzzy value is $\pi_{x_{3}} \neq 0, \pi_{y_{3}} \neq 0$., which means X3 and Y3 have certain uncertainty, so it is unreasonable for their distance to be 0 , instead, the reasonable value should be equal to certain nonzero number.

$d_{i}\left(x_{5}, y_{5}\right)=0,(i=1,2, \mathrm{~L}, 9)$, as $x_{5}=\left\langle x_{5}, 0,0\right\rangle, y_{5}=\left\langle y_{5}, 0,0\right\rangle$, they are special intuitionistic fuzzy values, characterized in that not only their membership degree is zero, but also the nonmembership degree is also zero, while the hesitancy degree is the maximal value of 1 . People are completely ignorant of such information. It is absurd for their distance to be zero, and their distance should be at least not equal to 0 , reasonably should be equal to a nonzero number not greater than 1 .

\section{New Definition and Formula of Distance Measure of Intuitionistic Fuzzy Set}


Based on above analysis, the new definition of distance measure of intuitionistic fuzzy set should emphatically solve: when hesitancy degree is not zero, the distance measure of two equal intuitionistic fuzzy values should not be zero. Restore property $(P 4)$ into triangle inequality of classical distance measure to get:

Definition 5 Set $A, B, C \in \operatorname{IFS}(X)$, mapping $D: \operatorname{IFS}(X) \times \operatorname{IFS}(X) \rightarrow[0,1]$ is called the distance measure between intuitionistic fuzzy sets $\mathrm{A}$ and $\mathrm{B}$, if it meets following Criterion:

(C1) $D(A, B)=D(B, A)$;

(C2) $D(A, B)=0$ if and only if $A=B$ and $\pi_{A}\left(x_{i}\right)=0, \pi_{B}\left(x_{i}\right)=0, i=1,2, \cdots \quad$;

(C3) $0 \leq D(A, B) \leq 1$;

(C4) $D(A, C) \leq D(A, B)+D(B, C)$.

Theorem 1 Set $x=\langle x, \mu(x), v(x)\rangle, y=\langle y, \mu(y), v(y)\rangle$ as intuitionistic fuzzy value, then following formula is the distance measure between intuitionistic fuzzy values $x$ and $y m=0,1,2, \cdots$ :

$$
D^{(m)}(x, y)=\frac{3\left|\mu^{(m)}(x)-\mu^{(m)}(y)\right|+3\left|v^{(m)}(x)-v^{(m)}(y)\right|+3\left|S^{(m)}(x)-S^{(m)}(y)\right|+2 \pi^{(m)}(x)+2 \pi^{(m)}(y)}{12}
$$

Example 2 If formula (6) is applied, and parameter $\mathrm{m}=2$, calculate the distance between the intuitionistic values $x j$ and yj $(i=1,2, \ldots 5)$. For common intuitionistic fuzzy value and fuzzy value, $D^{(2)}\left(x_{j}, y_{j}\right)=0, j=1,2,3$. While for true intuitionistic fuzzy value and special intuitionistic fuzzy value $D^{(2)}\left(x_{4}, y_{4}\right)=0.1 \neq 0 ; D^{(2)}\left(x_{5}, y_{5}\right)=\frac{1}{3} \neq 0$, these results embody the idea of definition 5 .

Similar to definition 5, the definition of distance of intuitionistic fuzzy set and definition of weighed distance of intuitionistic fuzzy set can be given. And similar to theorem 1, following theorems 2 and 3 can be obtained:

Theorem 2 Set $A=\left\{\left\langle x_{i}, \mu_{A}\left(x_{i}\right), v_{A}\left(x_{i}\right): x_{i} \in X\right\rangle\right\} \quad, B=\left\{\left\langle x_{i}, \mu_{B}\left(x_{i}\right), v_{B}\left(x_{i}\right): x_{i} \in X\right\rangle\right\}$ represents intuitionistic fuzzy set on limited domain of discourse $X$. Following formulas $D^{(m)}(E, C)$ is the distance between intuitionistic fuzzy sets $\mathrm{E}$ and $\mathrm{C}(m=0,1,2, \cdots)$ :

$$
D^{(m)}(E, C)=\frac{1}{12 n} \sum_{i=1}^{n}\left(3\left|\mu_{E}^{(m)}\left(x_{i}\right)-\mu_{C}^{(m)}\left(x_{i}\right)\right|+3\left|v_{E}^{(m)}\left(x_{i}\right)-v_{C}^{(m)}\left(x_{i}\right)\right|+3\left|S_{E}^{(m)}\left(x_{i}\right)-S_{C}^{(m)}\left(x_{i}\right)\right|+2 \pi_{E}^{(m)}\left(x_{i}\right)+2 \pi_{C}^{(m)}\left(x_{i}\right)\right)
$$

Theorem 3: Following formula $W D(E, C)$ is the weighed distance between intuitionistic fuzzy sets $\mathrm{E}$ and $\mathrm{C}(m=0,1,2, \cdots)$ :

$$
W D^{(m)}(E, C)=\frac{1}{12} \sum_{i=1}^{n} \omega_{i}\left(3\left|\mu_{E}^{(m)}\left(x_{i}\right)-\mu_{C}^{(m)}\left(x_{i}\right)\right|+3\left|v_{E}^{(m)}\left(x_{i}\right)-v_{C}^{(m)}\left(x_{i}\right)\right|+3\left|S_{E}^{(m)}\left(x_{i}\right)-S_{C}^{(m)}\left(x_{i}\right)\right|+2 \pi_{E}^{(m)}\left(x_{i}\right)+2 \pi_{C}^{(m)}\left(x_{i}\right)\right)
$$

Where the weight of element $x_{i}$ is $\omega_{i}\left(0 \leq \omega_{i} \leq 1\right)$ 且 $\sum_{i=1}^{n} \omega_{i}=1$

So-called building intuitionistic fuzzy set environment is to translate the raw data into intuitionistic fuzzy set data. Literature [14] presents the formula translating non-negative single value data into intuitionistic fuzzy set data, which takes the following form in this example:

$$
x_{i j}=\left\langle x_{i j}, \mu\left(x_{i j}\right), v\left(x_{i j}\right\rangle=\left\langle x_{i j}, \frac{x_{i j}}{x_{j \max }},\left(\frac{x_{i j}}{x_{j \max }}\right)^{\frac{1}{2}}\right\rangle\right.
$$

Where $x_{j \max }=\max \left\{x_{j 1}, x_{j 2}, x_{j 3}, x_{j 4}, x_{j 5}, x_{j 6}, x_{j 7}, x_{j 8}\right\}, j=1,2,3,4$.

\section{Summary}


The paper presented Vague set pattern recognition method based on intuitionistic fuzzy set distance and got effective distance data formula to be applied to the research on precise indoor ranging and positioning UWB technology in ship, improving the positioning accuracy and system capacity. While network expandability is to overcome geographical limitation of single network and realize precise indoor UWB RTLS centimeter-level positioning with large scope and high rate. Subsequently, the research needs to further combine the theory with the practice to get the precise wireless clock synchronization algorithm conforming to IEEE 802.15.4a UWB to be applied to Hainan EVK 2.0 hardware platform. For the target in single region positioning network, the clock synchronization between base stations is realized by realizing clock synchronization between master base station and slave base station via Kallman filtering. Meanwhile the laying becomes more convenient and maintenance cost for nodes is cut through wireless mode. The precise measurement data obtained by Vague set pattern recognition method of intuitionistic fuzzy set distance is applied to OMNET++ network simulation platform to verify the performance of related algorithms, and simulation serves as an auxiliary means to combine hainan EVK 1.0 in practice and conduct subsequently hardware platform test and implementation, thereby not only realizing scene application technology for indoor positioning in ship, but also providing an actual measurement platform for real-time precise positioning application scenes with large-scale label and big data flow in broad scope.

\section{Acknowledgement}

This research was financially supported by the National Natural Science Foundation of China (No.61461017); the Hainan Natural Science Foundation Innovation Research Team Project (No.2017CXTD0004); the Hainan Province Key Research and Development Projects (No.ZDYF2016002); the Innovative Research Project of Postgraduates in Hainan Province (No.Hyb2017-07).

\section{References}

[1] H. Suk-seung, K. Goo-Rak. AOA Selection Algorithm for Multiple GPS Signals, Asilomar Conference on Signals, Systems and Computers Proceedings, Pacific Grove, Nov 3-6, 2013, pp.48-485.

[2] G. Brian. Considerations and Challenges in Real Time Locating Systems Design, DecaWave White Paper, Dublin, 2008.

[3] L.Chung-Hsin. The Study for the RFID with Bluetooth Positioning System, 3rd International Conference on Information Sciences and Interaction Sciences Proceedings, pp.386-391, Chengdu, China, June 23-25, 2010.

[4] K.T. Atanassov. Intuitionistic fuzzy sets, Fuzzy Sets and Systems, 1986, 20(1):87-96.

[5] W.L. Gao, D.J. Buehrer. Vague Sets, IEEE. Transactions on Systems, Man, Cybernetics, 1993, 23(2):610-614.

[6] L.A. Zadeh. Fuzzy sets, Information and Control, 1965, (8): 338-353.

[7] H. Bustince, P. Burilbo. Vague sets are intuitionistic fuzzy sets, Fuzzy Sets and Systems, 1996, 79(3):403-405.

[8] G. Deschrijver, E.E. Kerre. On the relationship between some extensions of fuzzy set theory, Fuzzy Sets and Systems, 2003, 133(2): 227-235.

[9] E. Szmidt, J. Kacprzyk. Distances between intuitionistic fuzzy sets, Fuzzy Sets and Systems, 2000, 114(3):505-518. 
[10] Z. S. Xu. Some similarity measures of intuitionistic fuzzy sets and their applications to multiple attribute decision-Making, Fuzzy Optim Decis Making, 2007(6): 109-121

[11] H.X. Wang, F.J. Zhang, X.F.Fu. An Optimization Method Based on Vague Set Selection Architectural Engineering, 2012 International Conference on Industrial Control and Electronics Engineering, Xian, China, pp: 1254-1257.

[12] M.El. Hadidy, M.El. Absi, Artificial Diversity for UWB MB-OFDM Interference Alignment Based on Real-world Channel Models and Antenna Selection Techniques, IEEE International Conference on Ultra-Wideband Proceedings, Syracuse, Sept 17-20, 2012, pp: 338-342.

[13] E.I. Shah Saadon, J. Abdullah, Evaluating the IEEE 802.15.4a UWB physical layer for WSN applications, IEEE Symposium on Wireless Technology and Applications Proceedings, Sept 22-25, 2013, pp: 68-73,.

[14] Z.W. Li, X.M. Peng. Performance Evaluation of DC-OFDM System in China UWB Standard, International Conference on Computational Problem-Solving Proceedings, Chengdu, Oct 21-23, 2011, pp: 656-660.

[15] G.Ting, L.Wei. Flexible Analog Baseband for WiMedia MB-OFDM and China UWB Standard, 10th IEEE International Conference on Solid-State and Integrated Circuit Technology Proceedings, Shanghai, Nov 1-4, 2010, pp: 433-435.

[16] C. McElroy. Comparison of Wireless Clock Synchronization Algorithms for Indoor Location Systems, IEEE International Conference on Communications Workshops Proceedings, Sydney, June 10-14, 2014, pp: 157-162.

[17] C.Yin. Throughput Capacity of ALOHA MANETs, IEEE/CIC International Conference on Communications in China-Workshops Proceedings, Xi'an, Aug 12-14, 2013, pp: 71-75.

[18] S.Won-Yong, D.E.Lucani. Multi-Hop Routing is Order-Optimal in Underwater Extended Networks, IEEE International Symposium on Information Theory Proceedings Proceedings, June 13-18, Austin, 2010, pp: 510-514.

[19] J.Wentao, S.Limin. A Prediction Based Long-Cycle Time Synchronization Algorithm for Sensor Networks, Global Telecommunications Conference, Miami, Dec 6-10, 2010, pp: 1-5.

[20] L. Bin, R. Fengyuan, S. Junyang. Advanced Self-Correcting Time Synchronization in Wireless Sensor Networks, IEEE Communications Letters , 2010, pp:309-311.

[21] F.Kirsch, M.Vossiek, Distributed Kalman Filter for Precise and Robust Clock Synchronization in Wireless Networks, In Radio and Wireless Symposium, San Diego, Jan 18-22, 2009, pp: 482-485.

[22] K. Zhang, H.X. Wang, H.F. Wang, Z. Li. New Exploration on Definition of Similarity Measures Between Vague Sets, Journal of Natural Science of Heilongjiang University, 2012, 29(03): 412-415.

[23] K. Zhang, H.X. Wang, H.F. Wang, Z. Li. Vague Spatial Decision Method and Its an Application for the Location of Tailings Dam, Computer Science, 2014, 41(04): 260-262.

[24] K. Zhang, H.X. Wang, H.F. Wang, Z. Li. Park Water Quality Evaluation Method Based on Similarity Measures between Vague Sets, Computer Engineering \& Software, 2014, 35(02): 52-54. 